\title{
Understanding the Role of Arsenic in Camouflage Strategy: A Study with Common River Prawn
}

\author{
Mihieka Bose ${ }^{1}, \uparrow$, Kalpesh Jas ${ }^{1},+$, Arnab Barua ${ }^{2}{ }^{3}, *$ and Chayan Munshi ${ }^{4}, *$
}

1. Department of Zoology, Visva Bharati University, 731235, Santiniketan, India.

2. Departement de Biochimie, Université de Montréal, Québec, Canada

3. Centre Robert-Cedergren en Bio-informatique et Génomique, Université de Montréal, Québec, Canada

4. International Health Management Program, Berlin School of Business and Innovation, Potsdamer Straße 180-182, 10783 Berlin, Germany

$\dagger$ Shared first-author

* Corresponding author: Arnab Barua (arnab.barua@umontreal.ca), Chayan Munshi (chayanbio@gmail.com)

\begin{abstract}
Camouflage is an interesting adaptation (for survivability) by organisms in terms of different aggregation or fusion of colourations. Understanding these camouflage strategies in the presence of arsenic on transparent/semi-transparent species is pretty challenging. Previously, several researchers have demonstrated that colouration or pigmentation strategy in an organism is a strategy to merge with the environment to escape from predatory threats. Our study was done on a semi-transparent freshwater prawn species which exhibits a strategy of pigment droplets on its exoskeleton. Unlike previous studies, our findings robustly indicate the fact that pigment droplets are not the only reason for colouration. The pigment droplets rather regulate the darkness of the exoskeleton. However, the transparency of the abdominal muscles additionally plays a crucial role in creating a background of the pigment droplets. The transparency muscles allow light to pass through the abdomen, thus creating a semi-transparent appearance. The degree of semi-transparency is also regulated by the intensity of light. The abdomen and the pigment droplets on the exoskeleton cumulatively as well as contrastingly maintain the transparency and the colour quotient of the prawns. In our study, we have majorly concentrated on the abdominal region of the prawns as it appears to be the key semi-transparent feature of the organism. This research is directed to an ecotoxicological aspect where we showed that arsenic in chronic non-lethal concentration, can notably alter the colouration pattern in this prawn model within a short period of time. Finally, we have used an image processing algorithm to assess the alteration of colouration in this organism.
\end{abstract}


Keywords: Camouflage, Colouration, Arsenic, Toxicity, Common river prawn.

Introduction: Colouration in animals is genetically programmed and is regulated by several environmental factors. Carotenoids are the major molecules present in prawns that determine the colouration in the organisms ( $\mathrm{Su}$ et al., 2018). Our study is broadly based on analysing the colouration in a semi-transparent prawn model and the use of computational methods of assessing colour change in the organism by the influence of arsenic contamination. This perspective is rather a combination of ecotoxicological and evolutionary aspects of pigmentation in a semi-transparent aquatic arthropod.

Common river prawn is a semi-transparent species. However, broadly it is found in two coloured forms: the dark (black) and the light (white) coloured. The anterior part of the cephalothorax region of the body is observably darker than the abdominal area. The comparative darkness is somehow a result of the pigment droplets on the carapace (exoskeleton), the external surface of the body. The denser the pigment droplets, the darker it appears. There is a specific pattern of pigment deposition on the exoskeleton. A high accumulation of pigment droplets can be seen in every joint from head to tail as shown in Fig 1 on day 0 . The semi-transparency competently helps to camouflage the prawn in any background from the predator. However, the transparency can be altered by various biotic and abiotic stressors. Semi-transparent or transparent organisms are unique and can be effectively used as a model organism for visual tracking of the internal morphology and pigment distribution. Protocols for live imaging are much more convenient with semi-transparent species with sophisticated and appropriate but uncomplicated scientific devices. The process of camouflage is determined by the surrounding environment which has multiple backgrounds. Green and red chameleon prawns Hippolyte varians meticulously look like their seaweed substrates and can efficiently change their colour to camouflage with new backgrounds (Green et al., 2019). Whole-body transparency is a coherent camouflage strategy or approach that can be modified by several biotic and abiotic stressors. Tail-flip escape behaviour momentarily alters the transparency of the anemone shrimp Ancylomenes pedersoni and makes the abdominal muscle cloudy or opaque. Exercise upsurges the blood flow between muscles and generates low refractive index fluid between high refractive index muscles, which eventually enhance light scattering of light (Bagge et al., 2017). In the next section, we shall shed light on the camouflage strategy of prawns briefly. 


\section{Methods and results:}

Camouflage strategy: Studies show that pigmentation in crustaceans is determined by a range of genes and dietary carotenoids which are stabilised in the tissues by the protein crustacyanin $(\mathrm{CRCN})$. Experiments identify that $\mathrm{CRCN}$, sarcoplasmic calcium-binding protein and some types of actin may be involved in crustacean colouration (Ertl et al., 2013). When prawns are exposed to a light or dark coloured substrate, a colour adaptive response is generated which contracts or expands pigmentation structures in its hypodermis. This adaptive response cannot change the total pigment levels of carotenoid and astaxanthin. Colour in crustaceans changes due to physiological mechanisms such as carotenoid, background substrate colour, photoperiod, light intensity and temperature which can be rapid, reversible and rhythmic. These alterations are regulated by the expansion and contraction of pigment structure, known as chromatophore present in the hypodermal layer. Chromatophores are not solely responsible for colouration in prawns. Studies show that adaptive response cannot change the levels of the predominant carotenoid pigment, astaxanthin. Astaxanthin binds specifically to CRCN. Though no difference in CRCN gene expression is found in response to background substrate colour or moult cycle, the distribution of pigment in prawn hypodermal tissue is mainly regulated by CRCN protein (Wade et al., 2012). The pigmentation of prawns is highly influenced by factors like dietary carotenoid, hypodermal pigment dispersion and genetics. CRCN protein cannot be correlated with the prawn colouration as it does not control the alterations of colouration in response to the background colour (Wade et al., 2015). Under any biotic or abiotic stress, if muscle loses its translucency or gets clouded, the appearance of colouration also vicissitudes. Our experiments found that in high-intensity light prawn abdomen seems to be more transparent in water media (the natural habitat of prawns), as it can effortlessly cross abdominal muscles. The colouration of prawns primarily depends upon carotenoids and predominantly astaxanthin. Pigments are generally sited in the cephalothorax, abdominal epidermal layer and exoskeleton. A homogeneous dispersal of pigmentation is found in the epidermal layer of more coloured prawns than the concentrated pigment areas of light-coloured ones. The pigment widely abundant throughout the body is detected to be nonesterified astaxanthin (Tume et al., 2009). Colour polymorphism and colour change give a significant adaptive advantage in order to camouflage. It can be asymmetric among different morphs across habitats, depending upon the background-morph combination (Duarte et al., 2018). To better understand the colour change, we analysed the colour components of the prawn's abdominal part in the presence of Arsenic. 
Image analysis of prawn's abdominal part: A nifty information from a particular image can be interpreted by analysing it (Solomon et al., 2010). In this scenario, we use colour identification software (based on machine learning) in Python (Bhanot et al. 2018) to better reveal the colouration of prawns from the images of the prawn's abdomen. The colour extraction of the prawn's image is based on the KMeans algorithm and it is refined from the primary colours ( i.e., Red, Blue and Green ). Please note that we identified 5 major colours in the prawn's abdomen image using the KMeans algorithm for better comparison. Interestingly, we find that at the arsenic trioxide concentration at $1.7 \mathrm{ppm}$, the pigment droplets on the exoskeleton increased from day 0 to day 5 and later decreased on day 10. On the other hand, the skin colour or abdomen colour (which is semi-transparent) becomes darker from day 2 until day 5. Although the colour change until day 5 is debatable due to the influence of camouflage, water media and environmental constraints. Furthermore, on day 10, one can observe the whitish shade in the abdominal body part of the prawn, which is robust under every environmental restraint, as can be observed in Fig 1. Please note that, as we took the images of the prawn's abdominal body part with or without the presence of Arsenic, the ethical review and approval were waived off for this study.

Conclusions: Colouration in animals is genetically regulated by phenotypic expression, which has been established as a very significant marker of several biological events, including mate selection, camouflaging etc. Undoubtedly, pigmentation has an evolutionary impact and has been effectively studied in the broad area of behavioural ecology. However, semi-transparent or transparent organisms are the ones to manifest a complicated visual effect which is another form of concealment. In fact, aquatic semi-transparent organisms are reliable but complex models to unravel the mechanism of their colouration or camouflage in the aquatic environment. The strategy to hide from predators, escape or defend is taken by diverse organisms, predominantly to encounter the prey-predator interactive threats anytime. Our study is a novel approach to understanding the colouration pattern in a semi-transparent aquatic (freshwater) prawn model. From previous studies, it is well understood by several researchers that colouration in prawns occurs according to the sediment or background to merge completely with the same. Unlike coloured organisms, a semi-transparent or transparent organism does not effectively depend on the background colour to meld with, rather they have their own mechanism of concealment in an open environment. 

Day 0
Day 2
Day 4
Day 5
Day 10
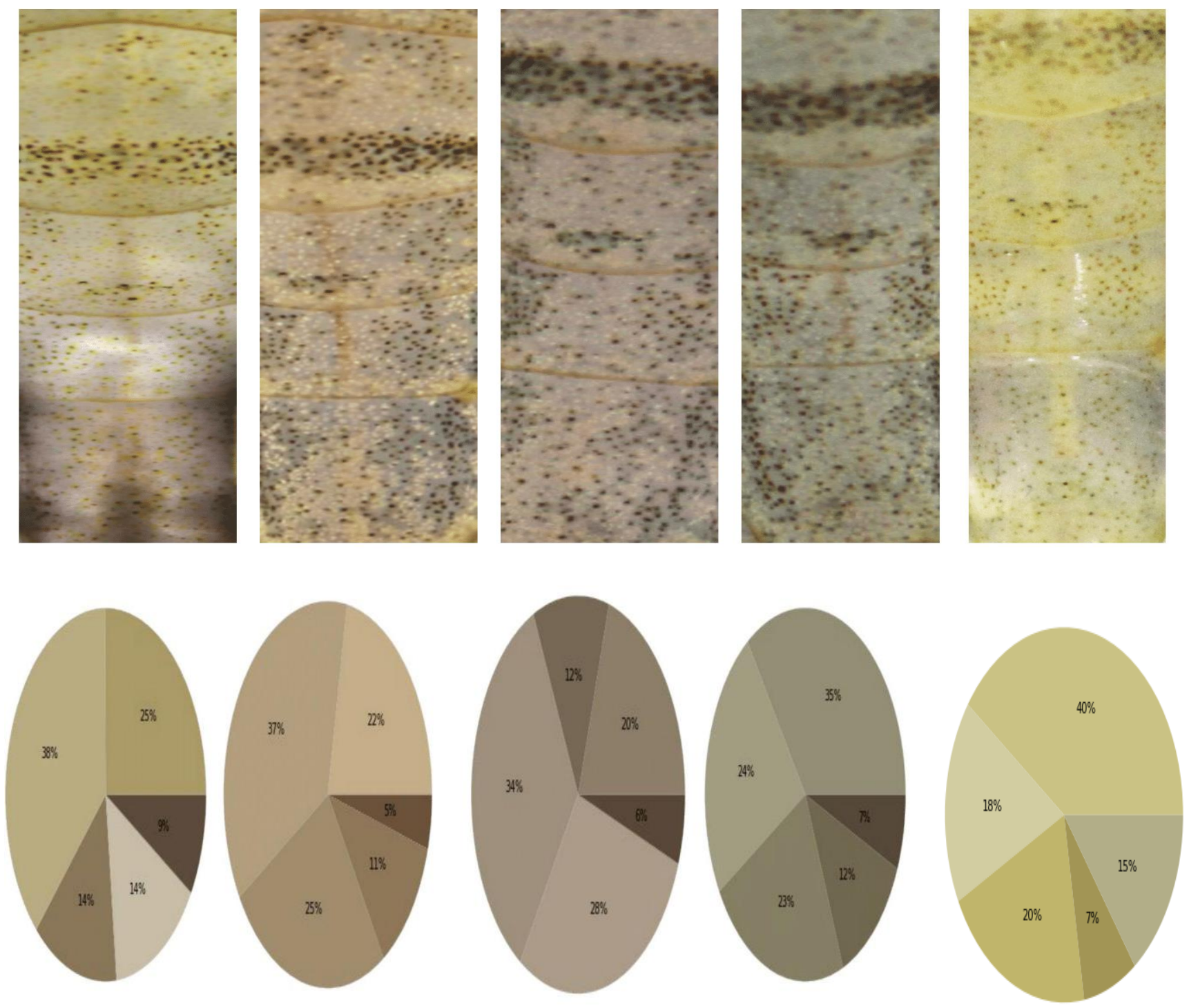

Fig 1: The image of the colouration of the prawn's abdominal body is taken from day 0 until day 10. The colour component of each image has been analysed using the KMeans algorithm, which shows how the shed of the colouration in prawns changed on a daily basis due to the presence of Arsenic in the environment.

This strategy efficiently makes these organisms evolutionarily successful. Chromatophores are widely recognised for their contribution to maintaining body colours. In our study model, we have found that despite the fact that our prawn model is semi-transparent, they have very prominent dark pigment spots or droplets on the body surface, which plays a crucial role in the formation of the dark colour. It is believed that the chromatophores are responsible for the formation of the pigment droplets on the body's exoskeleton. 
We focused on the prawn abdomen, as it was the most transparent part of the body. Initially, our main aim was dedicated to the pigment droplets on the surface and to assessing the darkness of the abdomen by qualifying their concentration. However, later through a series of observations it became evident that due to the semi-transparency the abdomen region can appear differently in different angles depending on the light intensity or the light angle (source). This is because light can easily cross the transparent abdominal muscles and create a glass-like appearance. The opaque the muscles (due to some specific physiological conditions), lesser the transparency. Our hypothesis strongly indicates that the light crosses the water and muscle media and eventually reaches the eyes of the predators ( as in Fig 1 ), which is definitely a reason for its transparency, but it has an effect on the pigment droplets scattered on the abdomen surface. Depending on the light intensity, the transparency alters, and this has a contrasting effect on the pigment darkness. The cumulative but apparently contrasting effects of the abdomen muscle and the pigments regulate the colour intensity (darkness) and transparency, where light intensity and angle is the parameter of concern. The same prawn on a fixed time frame can manifest diverse levels of transparencies and darkness. This is extremely unique in this kind of organism and for that reason, they are evolutionarily a unique example.

In our current framework, it will be also interesting to see how other toxic components and heavy metals can influence the camouflage strategy. Additionally, we portray a novel hypothesis that background colour is not enough to change the colour of a prawn, rather arsenic is an environmental toxicant that induces a change in the colour of prawns maintained without background. In fact, it is a toxicological approach shown in our study, that a non-lethal concentration (chronic dose) of arsenic trioxide can have a notable acute response, within 48 hours in terms of colour change. Contamination of arsenic is enough to induce the abdominal muscles and pigment droplets to alter within a short time span.

Author Contribution: Conceptualization, M.B., K.J., A.B. and C.M.; methodology, M.B., K.J., A.B. and C.M; software, A.B.; formal analysis, M.B., K.J., A.B. and C.M.; writing--original draft preparation, M.B., K.J., A.B. and C.M.; writing---review and editing, M.B., K.J., A.B. and C.M.; supervision, A.B. and C.M. All authors have read and agreed to the published version of the manuscript.

Funding: This research received no external funding. 
Institutional Review Board Statement: Not applicable. As we took the images of the prawn's abdominal body part with or without the presence of Arsenic only. So, ethical review and approval were waived off for this study.

Acknowledgements: The authors are grateful to the members of Ethophilia (an autonomous research group) [https://sites.google.com/view/ethophilia/home?authuser=0] for their constant support.

Conflicts of Interest: The authors declare no conflict of interest.

\section{References}

Tume et al. (2009). Effect of background colour on the distribution of astaxanthin in black tiger prawn (Penaeus monodon): Effective method for improvement of cooked colour. Aquaculture, 296(1-2), pp.129-135.

Bagge, L.E., Kinsey, S.T., Gladman, J. and Johnsen, S., 2017. Transparent anemone shrimp (Ancylomenes pedersoni) become opaque after exercise and physiological stress in correlation with increased hemolymph perfusion. Journal of Experimental Biology, 220(22), pp.42254233.

Ertl, N.G., Elizur, A., Brooks, P., Kuballa, A.V., Anderson, T.A. and Knibb, W.R., 2013. Molecular characterisation of colour formation in the prawn Fenneropenaeus merguiensis. PloS one, 8(2), p.e56920.

Green, S.D., Duarte, R.C., Kellett, E., Alagaratnam, N. and Stevens, M., 2019. Colour change and behavioural choice facilitate chameleon prawn camouflage against different seaweed backgrounds. Communications biology, 2(1), pp.1-10.

Wade, N.M., Anderson, M., Sellars, M.J., Tume, R.K., Preston, N.P. and Glencross, B.D., 2012. Mechanisms of colour adaptation in the prawn Penaeus monodon. Journal of Experimental Biology, 215(2), pp.343-350.

Duarte, R.C., Stevens, M. and Flores, A.A.V., 2018. The adaptive value of camouflage and colour change in a polymorphic prawn. Scientific reports, 8(1), pp.1-10. 
Wade, N.M., Budd, A., Irvin, S. and Glencross, B.D., 2015. The combined effects of diet, environment and genetics on pigmentation in the giant tiger prawn, Penaeus monodon. Aquaculture, 449, pp.78-86.

Su, F., Huang, B. and Liu, J., 2018. The carotenoids of shrimps (Decapoda: Caridea and Dendrobranchiata) cultured in China. Journal of Crustacean Biology, 38(5), pp.523-530.

Solomon, C.J., Breckon, T.P. (2010). Fundamentals of Digital Image Processing: A Practical Approach with Examples in Matlab. Wiley-Blackwell.

Bhanot et al. (2018). Color Identification in Images Machine Learning Application. Towards data science 